\title{
Quantization and Analysis of Toll Plaza Based on Probability Distributions and Queuing Theory
}

\author{
Feng Zhang \\ North China Electric Power University, Baoding 071000, China \\ 445324940@qq.com
}

Keywords: toll plaza; probability distributions; quantification model

Abstract. The toll station, served as the bond to highways, has always been the bottleneck to the capacity of the highway. Based on probability distributions and queuing theory, the thesis builds the quantitative model of capacity and calculates two evaluation indicators to analyze the drawbacks of the traditional toll station.

\section{Introduction}

The toll station, served as the bond to highways, has always been the bottleneck to the capacity of the highway. As for traditional toll stations, consider a toll highway with L lanes of travel in each direction and a barrier toll containing $n$ tollbooths in each direction. This thesis builds models to settle problems of modeling and making quantifications of traditional toll plaza.

\section{The Probability Distribution Model}

\section{The Distribution of the Vehicle Arrival.}

When the traffic is light, the vehicles are free to overtake and the headway changes frequently in a large scale. When the traffic is heavy, vehicles are constrained by other vehicles with diminished opportunities of overtaking. The vehicles begin to queue and the headway tends to be stable. Hence, the distribution of the vehicle arrival at the toll station is basically the same as that of the headway on the expressway.

The headway approximately obeys the Poisson distribution:

$$
P(x)=e^{-\lambda t} \frac{(\lambda t)^{x}}{x !}
$$

\section{The Distribution of the Service Time.}

Service time is the time from when the vehicle enters the tollbooth until it leaves the toll booth. It obeys certain law of distribution.

$$
B(x)=\left\{\begin{array}{rr}
1-e^{-\mu x}, x \geq 0 \\
0, x<0
\end{array}\right.
$$

\section{The Quantification of Evaluation Indexes.}

The service level of the toll station determines the waiting time of the vehicle, which has a direct impact on its capacity. The thesis uses the average length of single queue $(\bar{L})$, average number of vehicles in a tollbooth $(\bar{N})$,the average waiting time of the vehicle $(\bar{\omega})$ to evaluate the service level at toll stations. The specific calculation method is as follows ${ }^{[1]}$ :

$$
\left\{\begin{array}{c}
\bar{L}=\frac{\rho(k \rho)^{k}}{k !(1-\rho)^{2}} P_{0} \\
\bar{N}=\frac{\rho(k \rho)^{k}}{k !(1-\rho)^{2}} P_{0}+k \rho \\
\bar{\omega}=\frac{\rho(k \rho)^{k}}{\lambda k !(1-\rho)^{2}} P_{0}
\end{array}\right.
$$




$$
\begin{aligned}
& P_{0}=\frac{1}{\sum_{n=0}^{k-1} \frac{(\rho k)^{n}}{n !}+\frac{(\rho k)^{k}}{k !(1-\rho)}} \\
& \rho=\frac{\lambda}{k \mu}
\end{aligned}
$$

The Queuing Theory.

In the absence of any constraints, when the vehicle arrives at the toll plaza, the driver chooses a certain toll gate to enter. The basis of choice may be the closer distance, less vehicles at the toll gate, the tendency for the central gate, etc. Based on these factors, the article gives an entry probability for each toll gate, which means that different toll gates have different priorities for random selection. Hence, calculate the formula (3),(4),(5) and get the indicators for the k-th toll lane:

1 . Average number of vehicles in the $\mathrm{k}$-th lane

$$
\begin{aligned}
& \overline{N^{(k)}}=\frac{\rho_{k}}{1-\rho_{k}} \\
& \rho_{k}=\frac{C_{k} \lambda}{\mu}
\end{aligned}
$$

Only one car is charged while other vehicles are waiting in the queue. So the average queue length of the lane is:

$$
\overline{L^{(k)}}=\frac{\rho_{k}{ }^{2}}{1-\rho_{k}}
$$

2. The average waiting time of the k-th lane

$$
\overline{\omega^{(k)}}=\frac{\rho_{k}}{\left(1-\rho_{k}\right) \mu}
$$

\section{The Judgments of the Two Important Precautions}

\section{Accident Prevention.}

The thesis uses the accident data and road design of Baqiao and Lintong toll stations in Xi'an ${ }^{[2]}$. Then carry out a regression analysis of the road design parameters and the number of accidents and get the regression equation of road design parameter and number of annual accidents to predict the number of accidents of different toll stations in different road design parameters.

The regression equation is obtained by regression calculation from the sample data:

$$
Y=-4.4012-9.9475 x_{1}+10.0988 x_{2}+11.25 x_{3}
$$

Where,

$Y$ is the number of annual accidents;

$x_{1}$ is the gradient rate of the toll plaza;

$x_{2}$ is the service level of the toll plaza;

$x_{3}$ is the longitudinal slope at the entrance of the toll plaza.

\section{Throughput.}

On the basis of the previous analysis we know that when the vehicles don't line up at the toll station, all the vehicles that arrive can pass through the toll station quickly, and the throughput depends on the arrival of the vehicles. That is:

$$
C_{\text {out }}=C_{\text {in }}
$$

$C_{\text {out }}$ is the throughput of single toll lane;

$C_{i n}$ is the vehicle arrivals of single toll lane.

When the vehicles line up at the toll station, the vehicles cannot pass through the toll station 
timely and the throughput depends on the service time of the toll gate:

$$
\begin{gathered}
C_{\text {out }}=\frac{3600}{E(t)} \\
\mu=\frac{C_{\text {out }}}{3600}
\end{gathered}
$$

Hence, due to the different methods of collection and the different degrees of automation, different toll stations have different service time.

Table 1 The service time of various types of toll system

\begin{tabular}{ccc}
\hline Types of toll system & Mathematical expectation for service time[s] & $\begin{array}{c}\text { The throughput } \\
\text { [pcu/h] }\end{array}$ \\
\hline Human-staffed & 8 & 450 \\
\hline Exact-change & 6.2 & 580 \\
\hline Electronic toll & 2.25 & 1600 \\
\hline
\end{tabular}

\section{Actual Calculation and Analysis}

The calculation of the road capacity.

Take Xi'an Lintong toll stations for an example. It is human-staffed and the traffic volume of three traffic lanes is $655 p c u / h, 626 p c u / h$ and $602 p c u / h$. Given that drivers choose the lane independently, each toll gate has an entry probability. Search data, and get the entry probabilities of eight toll gates.

Table 2 The entry probabilities of toll gates

\begin{tabular}{ccccccccc}
\hline Gate number & 1 & 2 & 3 & 4 & 5 & 6 & 7 & 8 \\
\hline $\begin{array}{c}\text { Entry } \\
\text { probability }\end{array}$ & 0.306 & 0.212 & 0.167 & 0.149 & 0.111 & 0.083 & 0.056 & 0.028 \\
\hline
\end{tabular}

From the table above, the driver tends to drive without lane change, so the number of vehicles passing through each toll gate has a normal distribution. Therefore, for each of the three entry lanes, there is a difference in the traffic distribution.

Under the premise that the traffic flow of each traffic lane is known, the traffic volume of each toll lane can be obtained.

$$
\left(\begin{array}{l}
C_{i n 1} \\
C_{i n 2} \\
C_{i n 3} \\
C_{i n 4} \\
C_{i n 5} \\
C_{i n 6} \\
C_{i n 7} \\
C_{i n 8}
\end{array}\right)=\left(\begin{array}{lll}
0.306 & 0.212 & 0.167 \\
0.212 & 0.306 & 0.212 \\
0.167 & 0.212 & 0.306 \\
0.149 & 0.167 & 0.212 \\
0.111 & 0.149 & 0.167 \\
0.083 & 0.111 & 0.149 \\
0.056 & 0.083 & 0.111 \\
0.028 & 0.056 & 0.083
\end{array}\right)\left(\begin{array}{l}
655 \\
626 \\
602
\end{array}\right)=\left(\begin{array}{l}
458 \\
433 \\
426 \\
329 \\
266 \\
213 \\
155 \\
103
\end{array}\right)
$$

As is shown, the congestion degree of the $1^{\text {st }}$ toll gate is the largest, while the congestion degree of other gates is relatively small. Through Table 1 , we can see the average service time for human-staffed tollbooths is 8 seconds. Use the equation 13 and get the average departure rate of the lane.

$$
\mu=450 / 3600=0.125
$$

Use the equation 6,9 to get the values of indicators for service level for each toll lane: 
Table 3 The values of indicators for service level

\begin{tabular}{ccccccccc}
\hline Gate number & 1 & 2 & 3 & 4 & 5 & 6 & 7 & 8 \\
\hline $\begin{array}{c}\text { Average number of } \\
\text { vehicles[unit] }\end{array}$ & 4.09 & 3.19 & 2.98 & 1.37 & 0.88 & 0.59 & 0.37 & 0.22 \\
\hline Average waiting time[s] & 32.69 & 25.55 & 23.87 & 10.93 & 7.06 & 4.74 & 2.99 & 1.76 \\
\hline
\end{tabular}

\section{The analysis of the three important precautions}

\section{a. Accident prevention}

The range of the gradient rate of the toll $\operatorname{plaza}\left(x_{1}\right)$ is $(1 / 10,1 / 7)$. The longitudinal slope at the entrance of the toll plaza is within the design range or fluctuating from $1 \%$ to $2 \%$. The range of the service level of the toll plaza $\left(x_{2}\right)$ is $(0.7,0.9)$.

Therefore, as for the traditional toll stations, the best way to prevent accidents is increasing the service level as well as reducing the waiting time.

b. The throughput

The station the thesis discusses is human-staffed. So when the vehicles line up, the throughput of the toll lane depends on the mathematical expectation of service time. When the vehicles don't line up, the throughput of the toll lane equals the vehicle arrivals. Classify the 8 toll gates of the toll station and get the total throughput of the toll station.

Table 4 The total throughput of the toll station

\begin{tabular}{ccccccccc}
\hline Gate number & 1 & 2 & 3 & 4 & 5 & 6 & 7 & 8 \\
\hline Queue or not & Yes & Yes & Yes & No & No & No & No & No \\
\hline The throughput & 450 & 450 & 450 & 329 & 266 & 213 & 155 & 103 \\
\hline Total & & \multicolumn{8}{c}{2416} \\
\hline
\end{tabular}

\section{Conclusions}

By the analysis above, we know that in traditional toll plazas, different gate has different entry probability, which will lead to the lack of capacity. At the same time, it is also bad for accident preventions as well as throughput.

\section{References}

[1] Jun Xie. Study of Analysis and Evaluation for Expressway Capacity and Service Quality [D]. Xi'an: Chang'an University. 2007

[2] Min Zhang, Hong Chen, Xiaowu Wu, Safety Evaluation Model for Expressway Toll Stations [J] . China Safety Science Journal, 2009 (10):139-144 